\title{
Second line of treatment for HER2-positive gastric cancer: an evolving issue
}

\author{
Giandomenico Roviello ${ }^{1}$, Martina Catalano ${ }^{1}$, Alberto D’Angelo ${ }^{2}$, Valeria Emma Palmieri ${ }^{1}$ \\ ${ }^{1}$ Department of Health Sciences, University of Florence, Florence, Italy \\ ${ }^{2}$ Department of Biology and Biochemistry, University of Bath, Bath, England, United Kingdom
}

Key words: trastuzumab; paclitaxel; ramucirumab; second line; gastric cancer

Rep Pract Oncol Radiother 2021;26(2):316-317

\section{Dear Editor,}

We read with great interest the manuscript written by Makiyama et al [1]. In this paper, the authors reported outcome results of the randomized phase II study WJOG7112G (T-ACT Study). The study compared the efficacy of single agent paclitaxel to paclitaxel plus the continuous administration of trastuzumab beyond progression in HER2-positive advanced gastric cancer patients who progressed following trastuzumab first-line standard chemotherapy. A total of 91 patients were randomized; of these, 46 received paclitaxel while, in contrast, 45 received paclitaxel plus trastuzumab. The median progression-free survival (PFS) was 3.2 months in the paclitaxel arm and 3.7 months in the paclitaxel with trastuzumab, whereas the overall response rate (RR) was $32 \%$ in the paclitaxel arm and $33 \%$ in the paclitaxel with trastuzumab arm. Lastly, the median overall survival (OS) was 10 months for both arms. The authors concluded that the strategy of trastuzumab beyond progression failed to improve PFS in patients diagnosed with HER2-positive advanced gastric cancer.

To date, there is urgent need to identify the optimal second or further line of therapy for the treatment of metastatic GC $[2,3]$ patients. Ramucirumab is a monoclonal antibody against VEGFR2 [4] approved as the second or further line of treatment for the HER2-positive population. However, as discussed by the authors, a limited number of studies have described the efficacy of ramucirumab for this subgroup of patients so far. In 2019, De Vita et al. [5] evaluated the efficacy and safety of ramucirumab plus paclitaxel in a small subgroup of 20 patients affected by gastric cancer from the RAINBOW trial who were previously administered with trastuzumab. The authors observed a median PFS of 4.2 months, a RR of $45.0 \%$ and a median OS of 11.4 months (Tab. 1). Although a comparison between the studies is difficult due to the different clinical characteristics of evaluated patients and the source of the data (retrospective versus prospective), we must report a lower efficacy for the trastuzumab beyond progression strategy compared to "standard" second-line paclitaxel plus ramucirumab regimen. Moreover, a meta-analysis published in 2018 [6] showed that continuation of trastuzumab beyond first-line therapy added to second-line therapy did not prolong OS.

More recently, the antibody-drug conjugate named trastuzumab deruxtecan, consisting of trastuzumab plus a cleavable tetrapeptide-based linker and a cytotoxic topoisomerase I inhibitor, showed significant improvements in terms of clinical response and OS - compared with standard thera-

Address for correspondence: Giandomenico Roviello MD PhD, Department of Health Sciences, University of Florence, viale Pieraccini, 6, 50139, Florence, Italy; e-mail: giandomenicoroviello@hotmail.it 
Table 1. Main outcomes in patients with HER2 positive gastric cancer treated with second line trastuzumab beyond progression or paclitaxel with ramucirumab plus paclitaxel

\begin{tabular}{|l|l|c|c|c|c|c|}
\hline Study & Treatment & Number & $\begin{array}{c}\text { Response rate } \\
\text { (\%) }\end{array}$ & $\begin{array}{c}\text { PFS OS } \\
\text { (months) }\end{array}$ \\
\hline T-ACT & Trastuzumab beyond progression + paclitaxel & 45 & 33 & 3.7 & 10 \\
\hline RAINBOW & Paclitaxel + ramucirumab & 20 & 45 & 4.2 & 11.4 \\
\hline
\end{tabular}

OS - overall survival; PFS — progression-free survival

pies - in patients with HER2-positive advanced GC who progressed while receiving at least two previous therapies, including trastuzumab [7].

Therefore, we strongly agree with the statement of the authors to compare future clinical trial of new anti-HER2 agents with paclitaxel plus ramucirumab as the control arm. However, considering a potential crosstalk between HER-2 signalling and angiogenesis [8], we strongly encourage the possibility to perform future combination trials using anti-HER2 therapies plus ramucirumab-based regimens.

\section{Conflict of interest}

The authors declare that there are no conflicts of interest.

\section{Financial disclosure}

The authors declare that there is no financial interest.

\section{References}

1. Makiyama A, Sukawa Y, Kashiwada T, et al. Randomized, Phase II Study of Trastuzumab Beyond Progression in Patients With HER2-Positive Advanced Gastric or Gastroesophageal Junction Cancer:WJOG7112G (T-ACT Study). J Clin Oncol. 2020; 38(17): 1919-1927, doi: 10.1200/ JCO.19.03077, indexed in Pubmed: 32208960.
2. Roviello G, D'Angelo A, Roudi R, et al. Novel Agents in Heavily Pretreated Metastatic Gastric Cancer: More Shadows Than Lights. J Oncol. 2019; 2019: 5692317, doi:10.1155/2019/5692317, indexed in Pubmed: 31354820.

3. Roviello G, Fancelli S, Gatta Michelet MR, et al. TAS-102 in gastric cancer: Development and perspectives of a new biochemically modulated fluroropyrimidine drug combination. Crit Rev Oncol Hematol. 2020; 152: 102987, doi: 10.1016/j.critrevonc.2020.102987, indexed in Pubmed: 32485527.

4. Afshari F, Soleyman-Jahi S, Keshavarz-Fathi M, et al. The promising role of monoclonal antibodies for gastric cancer treatment. Immunotherapy. 2019; 11(4): 347-364, doi: 10.2217/imt-2018-0093, indexed in Pubmed: 30678552.

5. De Vita F, Borg C, Farina G, et al. Ramucirumab and paclitaxel in patients with gastric cancer and prior trastuzumab: subgroup analysis from RAINBOW study. Future Oncol. 2019; 15(23): 2723-2731, doi: 10.2217/fon-2019-0243, indexed in Pubmed: 31234645.

6. Ter Veer E, van den Ende T, Creemers A, et al. Continuation of trastuzumab beyond progression in HER2-positive advanced esophagogastric cancer: a meta-analysis. Acta Oncol. 2018; 57(12): 1599-1604, doi: 10.1080/0284186X. 2018.1503421, indexed in Pubmed: 30264641.

7. Shitara K, Bang YJ, Iwasa S, et al. DESTINY-Gastric01 Investigators. Trastuzumab Deruxtecan in Previously Treated HER2-Positive Gastric Cancer. N Engl J Med. 2020; 382(25): 2419-2430, doi: 10.1056/NEJMoa2004413, indexed in Pubmed: 32469182.

8. Alameddine RS, Otrock ZK, Awada A, et al. Crosstalk between HER2 signaling and angiogenesis in breast cancer: molecular basis, clinical applications and challenges. Curr Opin Oncol. 2013; 25(3): 313-324, doi: 10.1097/ CCO.0b013e32835ff362, indexed in Pubmed: 23518595. 